\title{
AIDS surveillance in Europe: Status at 30th June 1993
}

By 30th June 1993, a cumulative total of 88,663 AIDS cases had been reported in the twelve countries of the EC and 5,641 in the six COST countries. The cumulative number of cases reported by country ranges from just over 60 (Luxemburg) to over 17,000 (France, Italy and Spain). The latter three countries account for $71 \%$ of all cases reported in the EC. The highest cumulative rates per million population are observed in Spain (513), Switzerland (468), France (447) and Italy (308).

Adjustment for reporting delays leads to an estimate of 95,429 cumulative AIDS cases diagnosed in the EC by 30th June 1993.

The analysis of cases by disease category and period of diagnosis shows that $80 \%$ of the cases diagnosed in the first half of 1993 presented an opportunistic infection at AIDS diagnosis $(1.7 \%$ associated with Kaposi's sarcoma; $78.3 \%$ alone or associated with other diseases). During the same period of diagnosis, the proportion of cases presenting with Kaposi's sarcoma alone was $7.7 \%$. This proportion has been continuously decreasing since the beginning of the epidemic: $20.5 \%$ among cases diagnosed before 1986 .

The analysis of cases by sex shows a continuing increase in the proportion of female AIDS cases: of the cases diagnosed in 1991, $16.4 \%$ were female, whereas in the first half of 1993 this proportion was $18.3 \%$. A continuing increase in age at diagnosis is noted among both male and female adult/adolescent cases. Between 1991 and the first half of 1993, the proportion of cases aged 25 to 29 years decreased from $24.1 \%$ to $18.8 \%$ in males and from $33.9 \%$ to $29.1 \%$ in females, whereas the proportion aged 30 to 34 years increased from $26.4 \%$ to $28.7 \%$ and from $24.8 \%$ to $31.9 \%$ respectively.

The proportion of adult/adolescent cases diagnosed in the heterosexual contact group has increased progressively to reach $13.2 \%$ in the first half of 1993. Of these, women represent $49.8 \% \quad(322 / 646)$. The proportion of cases diagnosed in the haemophiliac and transfusion recipient groups continues to decrease.

A cumulative total of 1,681 paediatric cases (children under 13 years of age) was reported in the EC by 30th June 1993. Of these, $82.7 \%$ are related to mother-to-child transmission.

\section{European centre for the epidemiological monitoring of AIDS}

Hôpital national de Saint-Maurice F-94410 Saint-Maurice/France 